\title{
Near-Capacity Joint Channel Estimation and Three-Stage Turbo Detection for MIMO Systems
}

\author{
Peichang Zhang ${ }^{1}$, Sheng Chen ${ }^{1,2}$, and Lajos Hanzo ${ }^{1}$ \\ ${ }^{1}$ Electronics and Computer Science, University of Southampton, Southampton SO17 1BJ, U.K. \\ E-mails: \{pz3g09, sqc, lh\}@ecs.soton.ac.uk \\ ${ }^{2}$ Faculty of Engineering, King Abdulaziz University, Jeddah 21589, Saudi Arabia
}

\begin{abstract}
We propose a novel joint channel estimation and three-stage iterative detection/decoding scheme for near-capacity MIMO systems. In our scheme, as usual, the detected soft information is first exchanged a number of times within the inner turbo loop between the unity-rate-code (URC) decoder and the MIMO soft-demapper, and the information gleaned from the inner URC decoder is then iteratively exchanged with the outer decoder in the outer turbo loop. Our channel estimator however exploits the a posteriori information produced by the MIMO softdemapper to select a sufficient blocks of high-quality detected soft bits, and it is naturally embedded into the original iterative three-stage detection/decoding process, without introducing the costly iterative loop between the decision-directed channel estimator and the three-stage turbo detector/decoder. Hence, the computational complexity of our joint channel estimation and three-stage turbo detection is similar to that of the three-stage turbo detection/decoding scheme associated with the perfect CSI. Moreover, our reduced-complexity semi-blind scheme is capable of achieving the optimal maximum-likelihood turbo detection performance attained under the perfect CSI, with the same number of turbo iterations.
\end{abstract}

\section{INTRODUCTION}

Multiple-input multiple-output (MIMO) wireless communication systems [1]-[3] have attracted substantial attention due to their potential of providing spatial diversity and/or multiplexing gains. Non-coherent MIMO systems, which do not require channel state information (CSI), have found wideranging applications [4], [5]. However, non-coherent MIMO systems suffer from the classical $3 \mathrm{~dB}$ penalty in signal-tonoise ratio (SNR) and, moreover, their design freedom is also restricted [4], [6]. By contrast, coherent MIMO systems offer better performance and enjoy high degree of design flexibility in comparison to their non-coherent counterparts. The performance of a coherent MIMO system, however, heavily relies on the accuracy of CSI. Training-based channel estimators [7] are capable of obtaining accurate CSI estimate at the expense of significantly reducing the system's throughput and imposing high CSI estimation complexity, since employing a large number of training symbols is necessary. In sequel, we will refer to coherent MIMO simply as MIMO.

In attempt to circumvent the difficulties imposed by pure training-based channel estimation $(\mathrm{CE})$, powerful iterative detection/decoding schemes have been incorporated with the decision-directed channel estimator to form joint $\mathrm{CE}$ and

The financial supports of the UK EPSRC under the auspices of the ChinaUK Science Bridge and the India-UK Advanced Technology Centre, as well as of the EU Concerto project are gratefully acknowledged. turbo detection/decoding [8]-[12], where only a small number of training symbols are employed for generating an initial channel estimate. The initial turbo detection/decoding is then carried out based on the initial channel estimate. After the convergence of the turbo detector and decoder, the detected data are fed into the channel estimator for updating the channel estimate. The decision-directed channel estimator and the turbo detector/decoder iterate a number of times until the channel estimate converges. The turbo detector/decoder is capable of improving the reliability of the detected signals to assist the decision-directed channel estimator and likewise, more accurate channel estimates will result in increasingly more accurate turbo detector/decoder output.

To reduce the error propagation in the decision-directed CE caused by erroneous detected symbols, soft-decision aided channel estimators, which are also known as turbo $\mathrm{CE}$ schemes, exploit the soft estimates of the detected signals for $\mathrm{CE}$ and are more robust against the error propagation than the hard-decision aided CE schemes [13]. These softdecision aided CE schemes can naturally be incorporated with turbo detection/decoding schemes to form joint CE and turbo detection/decoding structures. For example, an iterative softdecision based CE scheme was proposed in [14], which feeds back the probabilities of the decisions from the equalizer to the channel estimator. In [15], a soft-input Kalman channel estimator and a weighted turbo recursive least square channel estimator were proposed, while a soft-decision aided iterative $\mathrm{CE}$ and symbol detection technique was proposed in [16].

All these existing joint $\mathrm{CE}$ and turbo detection/decoding structures have a number of limitations. Firstly, an extra iterative loop between the channel estimator and the turbo detector/decoder is introduced, which requires a number of iterations to converge and, therefore, increases the computational complexity of the receiver considerably. Secondly, the $\mathrm{CE}$ itself in all these schemes imposes an extremely high complexity. This is because the number of bits involved in a single interleaved frame of a turbo code is typically very large, and the number $\tau$ of the symbol vectors in a turbo coded frame is usually several thousands. All the existing schemes use the entire frame of the detected soft or hard bits for CE. Assume that the least squares channel estimate (LSCE) is employed. The complexity of the LSCE is well known in the order of $\tau^{3}$, $\mathcal{O}\left(\tau^{3}\right)$, which can be unacceptably high. Thirdly, at low SNR conditions, $20 \%$ to $50 \%$ of the detected bits are erroneous decisions and, even with soft-decision aided CE schemes, the 
error propagation can still be serious, which will degrade the achievable performance. Therefore, all these existing schemes cannot approach the optimal maximum-likelihood (ML) turbo detection performance associated with the perfect CSI.

Against this background, we propose a reduced-complexity joint $\mathrm{CE}$ and three-stage iterative detection/decoding structure for near-capacity MIMO systems [17]. As usual, only a very small number of training symbols are utilised for obtaining an initial LSCE. Our original contribution is twofold. Firstly, we propose a block-of-bits selection based soft-decision aided channel estimation (BBSB-SCE) scheme, which selects the high quality or more reliable detected blocks of bits based on the a posteriori information produced by the MIMO softdemapper within the original inner turbo loop of the unity-ratecode (URC) decoder and MIMO detector. This novel BBSBSCE produces two desired effects. Since it only utilises the high quality decisions in $\mathrm{CE}$, unlike all the existing softdecision aided channel estimators, it does not suffer from the same performance degradation caused by erroneous decisions. Moreover, the number of the high-quality blocks of bits used can be at least one order of the magnitude smaller than $\tau$ and, therefore, the computational complexity of the LSCE based on our BBSB-SCE scheme is reduced to several orders of the magnitude smaller than the estimator based on the entire detected data frame. Secondly, our channel estimator is naturally embedded in the original three-stage turbo detection/decoding process, and no extra iterative loop is required between the channel estimator and the three-stage MIMO detector/decoder. Therefore, the complexity of our joint BBSB-SCE and threestage turbo detector/decoder structure is similar to that of the original three-stage turbo detector/decoder scheme associated with the perfect CSI. We will demonstrate that our proposed semi-blind joint BBSB-SCE and three-stage turbo detection/decoding scheme is capable of fully exploiting the "turbo effects" to attain the optimal ML performance obtained by the idealised three-stage turbo detector/decoder receiver furnished with the perfect CSI, despite using only the same number of turbo iterations as the latter.

\section{Joint CE And Three-Stage Turbo Receiver}

We consider the MIMO system consisting of $M_{t}$ transmit antennas and $M_{r}$ receive antennas for communication in a frequency-flat Rayleigh fading environment.

\section{A. MIMO System Model}

The transmitter consists of the two-stage serial-concatenated outer recursive systematic code (RSC) encoder and inner URC encoder, followed by the MIMO $L$-QAM modulator, for achieving near-capacity performance [17]. Let $\mathbb{C}$ denote the field of complex numbers and BPS $=\log _{2}(L)$. The MIMO system model is expressed as

$$
\boldsymbol{y}(i)=\boldsymbol{H} \boldsymbol{s}(i)+\boldsymbol{v}(i),
$$

where $i$ denotes the symbol index, $\boldsymbol{H} \in \mathbb{C}^{M_{r} \times M_{t}}$ is the MIMO channel matrix, $s(i) \in \mathbb{C}^{M_{t} \times 1}$ represents the transmitted $L$-QAM symbol vector, and $\boldsymbol{y}(i) \in \mathbb{C}^{M_{r} \times 1}$ denotes the received signal vector, while $\boldsymbol{v}(i) \in \mathbb{C}^{M_{r} \times 1}$ represents the noise vector, whose elements obey the complex-valued zeromean Gaussian distribution of $\mathcal{C N}\left(0, N_{0}\right)$, with a variance of $N_{0} / 2$ per dimension. The system's SNR is defined as $\mathrm{SNR}=E_{s} / N_{0}$, where $E_{s}$ is the average symbol energy.

At the receiver, upon obtaining the a priori log-likelihood ratios (LLRs) $\left\{L_{a}\left(u_{k}\right)\right\}_{k=1}^{M_{t} \cdot \mathrm{BPS}}$ from the channel decoder, where $\left\{u_{k}\right\}_{k=1}^{M_{t} \text {. BPS }}$ indicate the corresponding bits that map to the symbol vector $s(i)$, the a posteriori LLRs produced by the ML MIMO soft-demapper ${ }^{1}$ are expressed as [18]

$$
L_{p}\left(u_{k}\right)=L_{p}(k)=\ln \frac{\sum_{\boldsymbol{s}^{n} \in\left\{\boldsymbol{s}_{u_{k}=1}\right\}} \exp \left(p_{n}\right)}{\sum_{\boldsymbol{s}^{n} \in\left\{\boldsymbol{s}_{u_{k}=0}\right\}} \exp \left(p_{n}\right)},
$$

where $\left\{\boldsymbol{s}_{u_{k}=1}\right\}$ and $\left\{\boldsymbol{s}_{u_{k}=0}\right\}$ represent the $L$-QAM symbol vector sets with the corresponding bit $u_{k}=1$ and $u_{k}=0$, respectively. The probability metrics $\left\{p_{n}\right\}_{n=1}^{L^{M_{t}}}$ for the possible $L$-QAM symbol vectors $\left\{s^{n}\right\}_{n=1}^{L^{M_{t}}}$ are given as

$$
p_{n}=-\frac{\left\|\boldsymbol{y}(i)-\boldsymbol{H} \boldsymbol{s}^{n}\right\|^{2}}{N_{0}}+\sum_{k=1}^{M_{t} \cdot \mathrm{BPS}} \tilde{u}_{k} L_{a}\left(u_{k}\right),
$$

where $\left\{\tilde{u}_{k}\right\}_{k=1}^{M_{t} \text {. BPS }}$ indicate the corresponding bits that map to the specific symbol vector $s^{n}$.

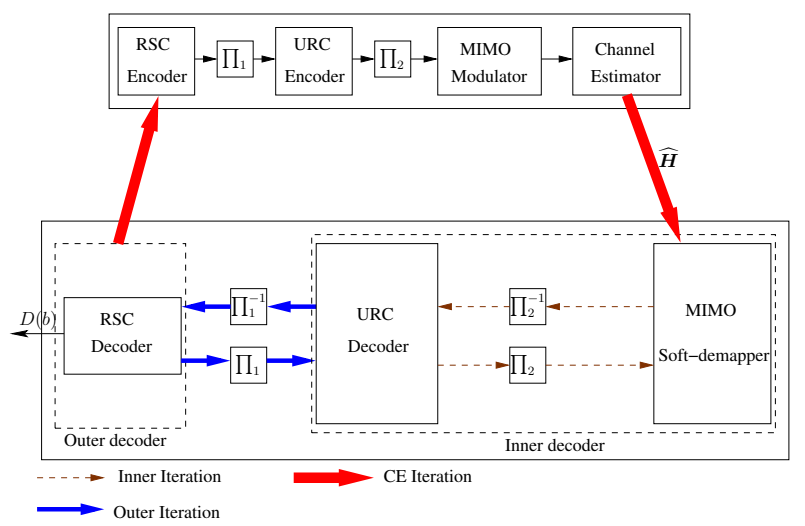

Fig. 1: Conventional joint channel estimation and three-stage turbo detector/decoder.

Let us first examine the conventional structure of joint $\mathrm{CE}$ and three-stage turbo detector/decoder as depicted in Fig. 1. To start the joint $\mathrm{CE}$ and three-stage turbo detector/decoder process requires an initial training based channel estimate. Assume that the number of the available training blocks is $M$ and the initial training data are arranged as

$$
\begin{aligned}
& \boldsymbol{Y}_{t M}=[\boldsymbol{y}(1) \boldsymbol{y}(2) \cdots \boldsymbol{y}(M)], \\
& \boldsymbol{S}_{t M}=[\boldsymbol{s}(1) \boldsymbol{s}(2) \cdots \boldsymbol{s}(M)] \text {. }
\end{aligned}
$$

Then the LSCE of the MIMO channel matrix $\boldsymbol{H}$ is given by

$$
\widehat{\boldsymbol{H}}_{L S C E}=\boldsymbol{Y}_{t M} \boldsymbol{S}_{t M}^{\mathrm{H}}\left(\boldsymbol{S}_{t M} \boldsymbol{S}_{t M}^{\mathrm{H}}\right)^{-1}
$$

${ }^{1}$ For large MIMO systems, we may opt for using near-optimum lowcomplexity detection schemes, e.g. the K-best sphere detector [19], [20], to avoid the exponentially increasing complexity imposed by the ML detector. 
where $(\cdot)^{\mathrm{H}}$ denotes the conjugate transpose operator. To maintain a high system throughput, only a small number of training blocks should be used. Note that to ensure a full rank of $\boldsymbol{S}_{t M} \boldsymbol{S}_{t M}^{\mathrm{H}}$, it is necessary to choose $M \geq M_{t}$. Therefore, $M_{t}$ is a lower bound for the number of initial training blocks. With $M$ chosen to be close to its lower bound $M_{t}$, the accuracy of the LSCE (6) is poor and hence the achievable bit error rate (BER) based on this initial LSCE is also poor. However, the three-stage turbo detector/decoder is capable of improving the reliability of the detected bits for assisting the soft decisiondirected channel estimator to provide a more accurate channel estimate, which in turn results in an increasingly more reliable turbo detector/decoder output.

Assume that, given the CSI, the two-stage inner turbo loop requires $I_{\text {inner }}$ iterations, while the outer turbo loop requires $I_{\text {outer }}$ iteration. Denote the complexity of the three-stage turbo detector/decoder as $C_{3 \mathrm{str}}\left(I_{\text {outer }} \cdot I_{\text {inner }}\right)$. Let a transmitted frame of the turbo coded bits contain $\tau$ symbol vectors, and further assume that the CE loop in Fig. 1 requires $I_{\text {ce }}$ iterations to converge. Then the complexity of the conventional joint $\mathrm{CE}$ and three-stage turbo receiver can be expressed as

$$
C_{\text {conventional }}=I_{\mathrm{ce}} \cdot \mathcal{O}\left(\tau^{3}\right)+I_{\mathrm{ce}} \cdot C_{3 \mathrm{str}}\left(I_{\text {outer }} \cdot I_{\text {inner }}\right),
$$

where $\mathcal{O}\left(\tau^{3}\right)$ is the complexity of the LSCE, as the entire frame of detected bits is used. Note that using the entire detected data frame leads to an extremely high CE complexity. More importantly, the frame of the detected bits may contain very large percentage of the erroneous decisions, particularly under low SNR conditions, which will degrade even the soft decision-directed channel estimator. Therefore, it is wellknown that there exists a BER performance gap between this semi-blind joint $\mathrm{CE}$ and three-stage turbo receiver and the idealised three-stage turbo receiver with the perfect CSI.

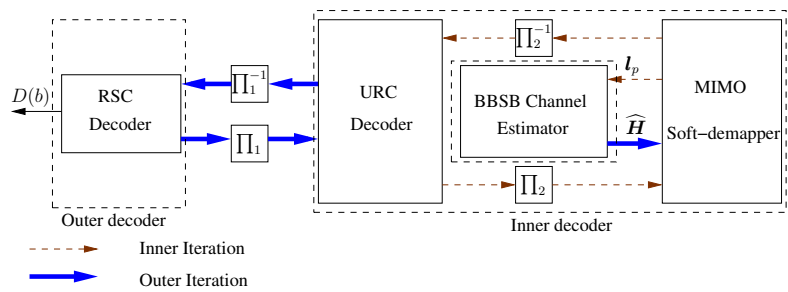

Fig. 2: Proposed joint BBSB-SCE and three-stage turbo detector/decoder.

\section{B. Joint BBSB-SCE and Three-Stage Turbo Receiver}

Our novel structure of semi-blind joint BBSB-SCE and three-stage turbo detector/decoder is depicted in Fig. 2. Notice the two significant differences with the conventional structure of Fig. 1. Firstly, there is no additional iterative loop involving the $\mathrm{CE}$ and the three-stage turbo detector/decoder. In other words, our soft decision aided channel estimator is embedded in the original outer loop of the three-stage turbo structure, and updating of the channel estimate occurs concurrently with the original outer turbo decoding iteration. Consequently, the complexity of our joint scheme is reduced to the same level of $C_{3 \text { str }}\left(I_{\text {outer }} \cdot I_{\text {inner }}\right)$. More importantly, our channel estimator does not use the entire frame of the detected bits. Rather, it only selects the high-quality decisions. Specifically, the $a$ posteriori information (2) output by the MIMO soft-demapper provides the confidence levels, i.e. the probabilities, of binary $1 \mathrm{~s}$ and 0s [17]. Therefore, based on this confidence level, we can select the reliable decisions from the MIMO softdemapper's output sequence for CE. Thus, the complexity of our soft-decision aided LSCE is dramatically lower than $\mathcal{O}\left(\tau^{3}\right)$. Moreover, as will be confirmed later in our simulation study, our joint BBSB-SCE and three-stage turbo receiver is capable of approaching the near-capacity optimal ML performance associated with the perfect CSI. With the notation

$$
\boldsymbol{Y}_{\mathrm{d} \tau}=[\boldsymbol{y}(1) \boldsymbol{y}(2) \cdots \boldsymbol{y}(\tau)]
$$

representing the MIMO observation data sequence, we now detail our semi-blind joint BBSB-SCE and three-stage turbo detector/decoder scheme.

Step 1) Set the outer turbo iteration index to $t=0$ and the initial channel estimate to $\widehat{\boldsymbol{H}}^{(t)}=\widehat{\boldsymbol{H}}_{L S C E}$.

Step 2) Given the channel estimate $\widehat{H}^{(t)}$, perform the ML MIMO soft-demapping based on the observation data $\boldsymbol{Y}_{\mathrm{d} \tau}$ of (8). The MIMO soft-demapper then exchanges its soft information with the URC inner decoder for $I_{\text {inner }}$ iterations. This yields the $I_{\text {inner }}$ vectors of the a posteriori information defined in (2), which can be arranged as

$$
\boldsymbol{L}_{p}=\left[\boldsymbol{l}_{p}^{1} \boldsymbol{l}_{p}^{2} \cdots \boldsymbol{l}_{p}^{I_{\mathrm{inner}}}\right]^{\mathrm{T}}
$$

where $(\cdot)^{\mathrm{T}}$ represents the transpose operator, $\boldsymbol{l}_{p}^{i} \in \mathbb{C}^{(\mathrm{BPB} \cdot \tau) \times 1}$ denotes the a posteriori information vector obtained by the MIMO soft-demapper during the $i$ th inner iteration, and $\mathrm{BPB}=M_{t} \cdot \mathrm{BPS}$, while $\boldsymbol{L}_{p} \in \mathbb{C}^{I_{\text {inner }} \times(\mathrm{BPB} \cdot \tau)}$ is the equivalent a posteriori information matrix. Based on the fact that the $n$th column of $\boldsymbol{L}_{p}$ represents the $I_{\text {inner }}$ soft decisions of the $n$th information bit, where $n \in\{1,2, \cdots,(\mathrm{BPB} \cdot \tau)\}$, the block of bits to be used for the CE is selected in either of the following two cases:

Case 1: If the soft decisions in the same column share similar values, these soft decisions may result in a relatively stable and reliable bit decision, which are hence invoked for the CE. Specifically, the criterion for the $n$th information bit to be selected is

$$
\frac{\left|L_{p}^{1}(n)-L_{p}^{2}(n)\right|+\cdots+\left|L_{p}^{I_{\text {inner }}-1}(n)-L_{p}^{I_{\text {inner }}}(n)\right|}{|\mu|} \in\left(0, T_{h}\right),
$$

where $\mu$ is the mean of the soft decisions in the $n$th column of $\boldsymbol{L}_{p}$, and $T_{h}$ denotes the block-of-bits selection threshold.

Case 2: If the absolute values of the soft decisions in the $n$th column appear to be in monotonically ascending order and share the same polarity (i.e. the decisions are all positive or negative), the $n$th information bit may be regarded as correct and hence is selected for the CE.

After checking through all the columns of $\boldsymbol{L}_{p}$, only high confidence decisions are selected and the corresponding symbol block indices can be obtained by a sliding-window method using a window size of BPB. More explicitly, if BPB consecutive 
information bits are all regarded as correct, the corresponding information block or symbol vector will be selected for the CE. This yields a high confidence integer-valued index vector, which is denoted as $\boldsymbol{x}^{t}=\left[x^{t}(1) x^{t}(2) \cdots x^{t}\left(\tau_{s}^{t}\right)\right]^{\mathrm{T}}$, with the number of the selected symbol vectors $\tau_{s}^{t}$ varying within ( $\left.0, \tau_{\text {sel }}\right]$, where $\tau_{\text {sel }} \ll \tau$ is the maximum number of blocks for the CE, imposed to limit the complexity of CE. By using this index vector, the corresponding observation data can be selected from (8), and re-arranged as

$$
\boldsymbol{Y}_{\mathrm{sel}}^{(t)}=\left[\boldsymbol{y}\left(x^{t}(1)\right) \boldsymbol{y}\left(x^{t}(2)\right) \cdots \boldsymbol{y}\left(x^{t}\left(\tau_{s}^{t}\right)\right)\right] .
$$

Step 3) Based on the selected high-confidence detected blocks of bits with the aid of the corresponding symbol vector indices $\boldsymbol{x}^{t}$, generate the soft-estimate of each symbol element as [21]

$$
\begin{aligned}
\widehat{s}^{m}\left(x^{t}(n)\right) & =\sum_{l=1}^{L} s^{l} \operatorname{Pr}\left\{s^{m}\left(x^{t}(n)\right)=s^{l}\right\} \\
& =\sum_{l=1}^{L} s^{l} \cdot \frac{\exp \left(\sum_{j=1}^{\mathrm{BPS}} \widetilde{u}_{j} L_{a}\left(u_{j}\right)\right)}{\prod_{j=1}^{\mathrm{BPS}}\left(1+\exp \left(L_{a}\left(u_{j}\right)\right)\right)},
\end{aligned}
$$

for $1 \leq n \leq \tau_{s}$, where $\left\{s^{l}\right\}_{l=1}^{L}$ denotes the $L$-QAM symbol set, $m \in\left\{1,2, \cdots, M_{t}\right\}$ indicates the symbol index in the soft-estimated symbol vector $\widehat{\boldsymbol{s}}\left(x^{t}(n)\right)$, and $\left\{\widetilde{u}_{j}\right\}_{j=1}^{\text {BPS }}$ represents the bit mapping corresponding to $\left\{s^{l}\right\}_{l=1}^{L}$. By arranging the soft-estimated symbol vectors as

$$
\widehat{\boldsymbol{S}}_{\mathrm{sel}}^{(t)}=\left[\widehat{\boldsymbol{s}}\left(x^{t}(1)\right) \widehat{\boldsymbol{s}}\left(x^{t}(2)\right) \cdots \widehat{\boldsymbol{s}}\left(x^{t}\left(\tau_{s}^{t}\right)\right)\right],
$$

the resulting decision-directed LSCE is given by

$$
\widehat{\boldsymbol{H}}^{(t+1)}=\boldsymbol{Y}_{\text {sel }}^{(t)}\left(\widehat{\boldsymbol{s}}_{\text {sel }}^{(t)}\right)^{\mathrm{H}}\left(\widehat{\boldsymbol{s}}_{\text {sel }}^{(t)}\left(\widehat{\boldsymbol{s}}_{\text {sel }}^{(t)}\right)^{\mathrm{H}}\right)^{-1} \text {. }
$$

This update occurs as the soft information is exchanged between the two-stage inner decoder and the outer RSC decoder. Step 4) Set $t=t+1$. If $t<I_{\text {outer }}$, repeat Steps 2) and 3); otherwise, stop.

The complexity of CE in our scheme is upper bounded by $\mathcal{O}\left(\tau_{\text {sel }}^{3}\right)$ which is much smaller than $\mathcal{O}\left(\tau^{3}\right)$. As an example, in the simulation we have $\tau=1000$ and we set $\tau_{\text {sel }}=100$. Therefore, the complexity of our channel estimator is more than 1000 times smaller than that of the conventional scheme. The total complexity of our proposed scheme is

$$
C_{\text {proposed }} \leq I_{\text {outer }} \cdot \mathcal{O}\left(\tau_{\text {sel }}^{3}\right)+C_{3 \text { str }}\left(I_{\text {outer }} \cdot I_{\text {inner }}\right) .
$$

Since the 1 st term is negligible in comparison with the 2 nd term, we have $C_{\text {proposed }} \approx C_{3 \text { str }}\left(I_{\text {outer }} \cdot I_{\text {inner }}\right)$.

\section{Simulation Results}

A quasi-static Rayleigh fading MIMO system with $M_{t}=4$, $M_{r}=4$ and 16-QAM was simulated. All the results were averaged over 100 channel realisations, which were generated with the normalised Doppler frequency of $f_{d}=0.01$. An interleaver length of 16,000 bits was used by the three-stage serial-concatenated turbo encoder/decoder. The binary generator polynomials of the RSC encoder were $G_{R S C}=[1,0,1]_{2}$ and $G_{R S C}^{r}=[1,1,1]_{2}$, while those of the URC encoder were $G_{U R C}=[1,0]_{2}$ and $G_{U R C}^{r}=[1,1]_{2}$, where $G_{R S C}^{r}$ and $G_{U R C}^{r}$ are the feedback polynomials of the RSC and URC encoders, respectively. The transmitted signal power was normalised to unity and, therefore, the SNR was given as $\frac{1}{N_{0}}$. The number of initial training data blocks was chosen to be $M=6$, while the maximum number of selected blocks for our BBSB-SCE was set to $\tau_{\text {sel }}=100$. Three metrics were used to assess the achievable performance, and they were the extrinsic information transfer (EXIT) chart [17], the BER and the mean square error (MSE) of the channel estimator. The Cramér-Rao lower bound (CRLB) [22], [23] is known to provide the best attainable performance for an unbiased estimator, and can be used to lower bound the MSE of a channel estimator.

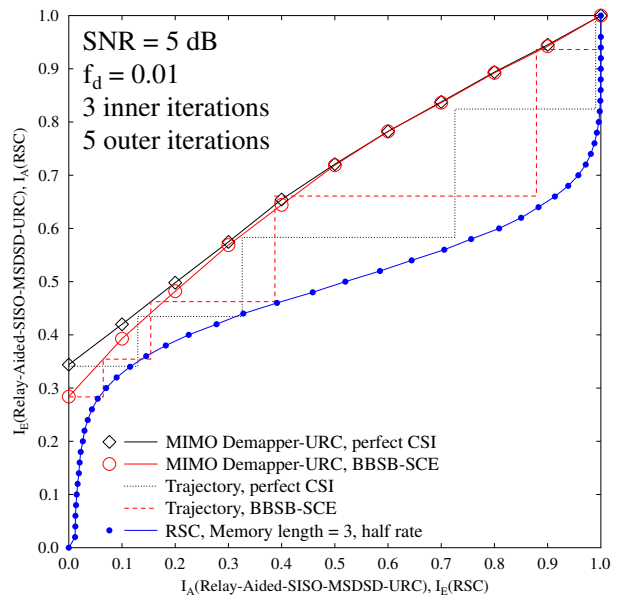

Fig. 3: EXIT chart analysis of our proposed semi-blind joint BBSBSCE and three-stage turbo receiver with the block-of-bits selection threshold of $T_{h}=1.0$, in comparison to the perfect-CSI scenario.

Fig. 3 shows the EXIT chart of our proposed joint BBSBSCE and three-stage turbo receiver with a selection threshold of $T_{h}=1.0$, in comparison to that of the perfect-CSI case. It can be seen that open tunnels exist between the EXIT curves of the amalgamated inner MIMO soft-demapper-URC decoder and the outer RSC decoder for both the proposed semi-blind BBSB-SCE based scheme and the idealised optimal ML detection based on the perfect CSI, at $\mathrm{SNR}=5.0 \mathrm{~dB}$. The Monte-Carlo simulation based stair-case shaped decoding trajectories, which closely match the EXIT curves, are also provided at $\mathrm{SNR}=5.0 \mathrm{~dB}$. It is seen that both of the trajectories are capable of reaching the perfect convergence point of $(1.0,1.0)$, implying that the proposed semi-blind BBSBSCE based scheme is capable of achieving the optimal ML detection performance with the same number of iterations. Additionally, it can also be seen that the starting point of the EXIT curve of the semi-blind BBSB-SCE based scheme is lower than that of perfect CSI scenario. This is obviously because poor initial CE quality leads to a lower extrinsic information. However, as the a priori information value increases, the EXIT curve of the BBSB-SCE based scheme is capable of converging to that of the perfect CSI scenario, implying that an accurate channel estimate has been obtained.

The BER performance of the proposed joint BBSB-SCE and three-stage turbo receiver is shown in Fig. 4, in comparison to 


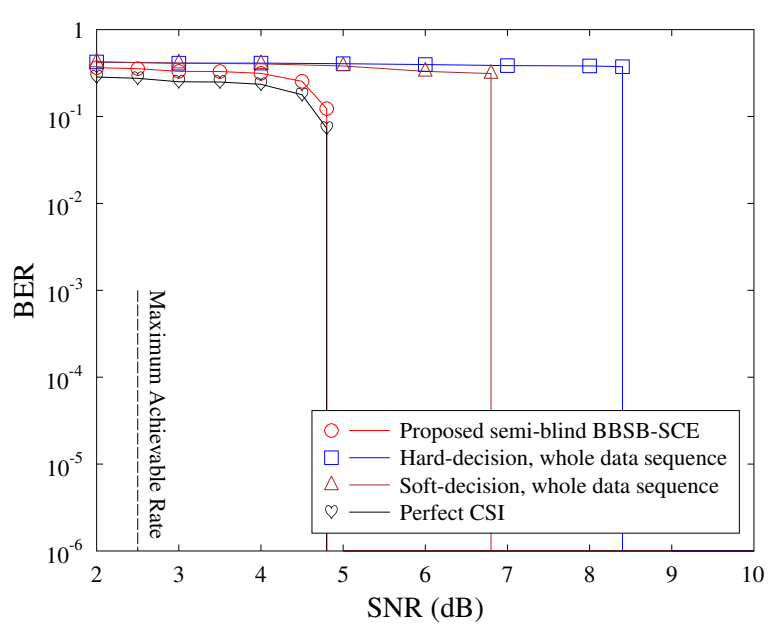

Fig. 4: BER performance of the proposed joint BBSB-SCE and three-stage turbo receiver with a block-of-bits selection threshold of $T_{h}=1.0$, in comparison to that of the perfect CSI scenario as well as those of the conventional joint $\mathrm{CE}$ and three-stage turbo receivers employing the entire detected data sequence for the soft-decision and hard-decision aided channel estimators, respectively.

that of the perfect CSI case as well as those of the conventional semi-blind joint $\mathrm{CE}$ and three-stage turbo schemes employing the soft-decision and hard-decision aided channel estimators, respectively, based on the entire detected data sequence. It can be seen that the proposed semi-blind BBSB-SCE based scheme is capable of converging to the near-capacity optimal ML performance associated with the perfect CSI, with the same "turbo-cliff" occurring at $\mathrm{SNR}=5 \mathrm{~dB}$ as predicted by the EXIT chart analysis of Fig. 3. Observe that the conventional joint $\mathrm{CE}$ and three-stage turbo receiver with the softdecision aided channel estimator employing the entire detected data sequence cannot attain the performance of the idealised optimal ML performance achieved under the perfect CSI, and there exists a $2 \mathrm{~dB}$ gap between the BER turbo-cliffs of the two receivers. This clearly demonstrates the power of only selecting high-quality blocks of bits for CE. The conventional scheme employing the hard-decision aided channel estimator

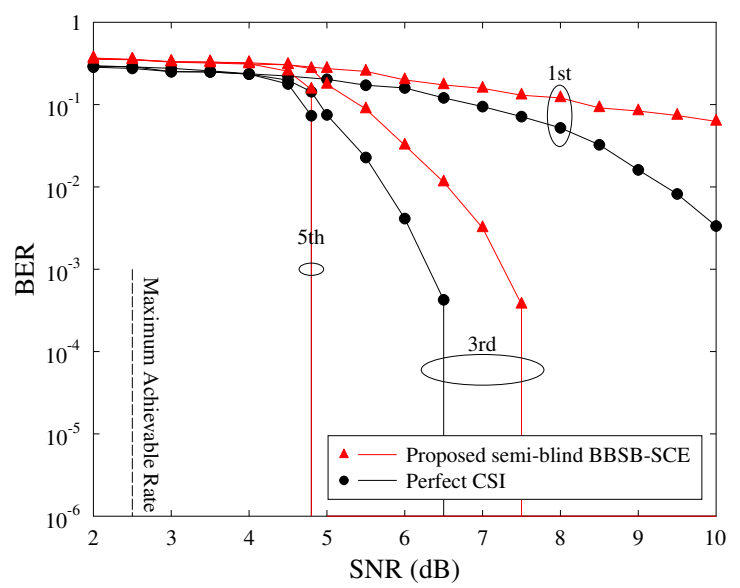

Fig. 5: BER convergence performance of the proposed joint BBSBSCE and three-stage turbo receiver with a block-of-bits selection threshold of $T_{h}=1.0$, in comparison to the perfect-CSI case. based on the entire detected data sequence exhibits further $1.5 \mathrm{~dB}$ degradation from its soft-decision assisted counterpart.

Fig. 5 illustrates the convergence behaviour of the proposed joint BBSB-SCE and three-stage turbo scheme, in comparison to that of the idealised three-stage turbo scheme based on the perfect CSI. Observe that for both the proposed semi-blind BBSB-SCE based and idealised three-stage turbo receivers, $I_{\text {outer }}=5$ outer iterations are sufficient to achieve the nearcapacity optimal performance. In addition, it can be seen that the BER gap between the proposed BBSB-SCE based scheme and the perfect CSI case reduces, as the number of outer iterations increases. Specifically, at the initial iteration, there exist a large BER gap, and during the third iteration, the BER gap is reduced to around $1 \mathrm{~dB}$, but at the fifth iteration, there exists no BER gap, indicating that the BBSB-SCE scheme has converged to the true MIMO channel.

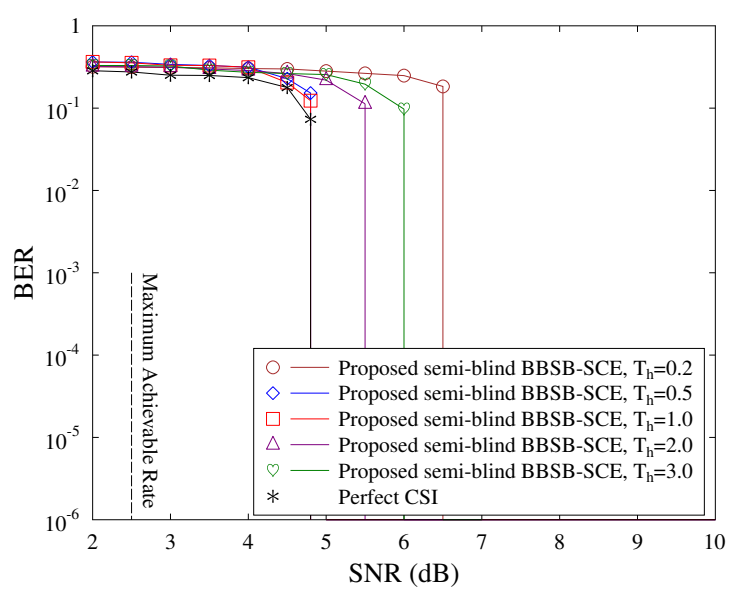

Fig. 6: Effects of the block-of-bits selection threshold $T_{h}$ on the BER performance of our proposed semi-blind joint BBSB-SCE and three-stage turbo receiver.

The effects of the block-of-bits selection threshold $T_{h}$ on the achievable BER performance of our proposed semiblind scheme were also investigated by varying the value of $T_{h}$ in the set $\{0.2,0.5,1.0,2.0,3.0\}$ under the same system configuration. The results obtained are shown in Fig. 6, where it is observed that for $T_{h}=0.5$ and 1.0, the BER performance of the proposed semi-blind joint BBSB-SCE and three-stage turbo scheme converges to that of the perfect-CSI case. However, for a relatively small threshold value of $T_{h}=0.2$, a performance degradation occurred, since the number of blocks of bits selected for the CE is probably insufficient. On the other hand, given relatively high values of $T_{h}=2.0$ and 3.0 , some unreliable decisions may have been selected for the $\mathrm{CE}$ and this can lead to a performance degradation from the perfect-CSI case, as can be seen from Fig. 6 . The results of Fig. 6 clearly demonstrate that as long as the threshold value is not chosen to be too small or too large, the performance of the semi-blind BBSB-SCE based iterative scheme is not too sensitive to the value of $T_{h}$ used. Indeed, there exists a wide range of values for $T_{h}$, which allow our scheme to approach the optimal performance of the perfect-CSI case even without increasing the number of turbo iterations. 


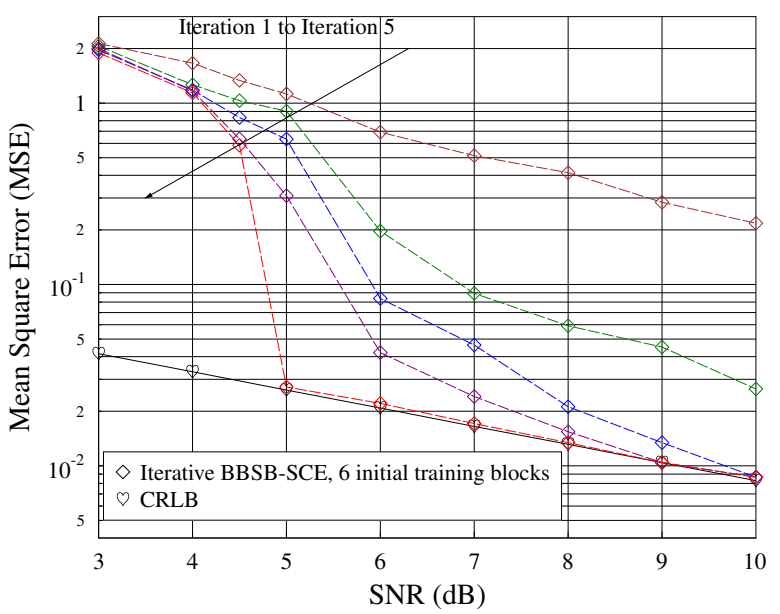

Fig. 7: MSE convergence performance of the channel estimator in our proposed semi-blind joint BBSB-SCE and three-stage turbo receiver using a block-of-bits selection threshold of $T_{h}=1.0$.

The MSE performance of the channel estimator in our proposed scheme is compared with the CRLB in Fig. 7, where it can be seen that the MSE of our decision-directed channel estimate approaches the CRLB, once the number of outer turbo iterations reaches $I_{\text {outer }}=5$ for $\mathrm{SNR} \geq 5.0 \mathrm{~dB}$. This corresponds to the BER cliff at $\mathrm{SNR} \approx 5.0 \mathrm{~dB}$ and $I_{\text {outer }}=5$ shown in Fig. 4, implying that the decision-directed channel estimator in our scheme is most efficient for $\mathrm{SNR} \geq 5.0 \mathrm{~dB}$, since it approaches the CRLB. However, it can also be seen that for $\mathrm{SNR}<5.0 \mathrm{~dB}$, the MSE of the channel estimate is degraded. This is expected because for such low SNR values, the open EXIT tunnel shown in Fig. 3 becomes closed and hence the BER becomes extremely large. Under such adverse conditions, a decision-directed channel estimator cannot be expected to approach the CRLB.

\section{CONCLUSIONS}

We have proposed a novel reduced-complexity joint BBSBSCE and three-stage turbo detection/decoding scheme for near-capacity MIMO systems. Unlike the existing methods, our scheme does not require an extra iterative loop between the channel estimator and the turbo detector/decoder, since our BBSB-SCE is naturally embedded in the original threestage demapping/decoding turbo loop. This novel arrangement enables us to reduce the computational complexity substantially. Most significantly, our BBSB-SCE scheme only selects high-confidence decisions for our soft-decision based channel estimate. This not only ensures that the complexity of our channel estimator is several orders of the magnitude smaller than the existing methods but also enables our proposed scheme to attain the near-capacity optimal ML performance of the idealised three-stage turbo receiver furnished with the perfect CSI, using the same low number of turbo iterations as the latter, as confirmed by our simulation results.

\section{REFERENCES}

[1] N. Chiurtu, B. Rimoldi, and E. Telatar "On the capacity of multi-antenna Gaussian channels," in Proc. 2001 IEEE Int. Symp. Information Theory (Washington, DC , USA), June 24-29, 2001, p. 53.
[2] B. Hassibi and B. M. Hochwald, "High-rate codes that are linear in space and time," IEEE Trans. Information Theory, vol. 48, no. 7, pp. 18041824, 2002.

[3] K.-C. Huang and Z. Wang, "Millimeter-wave circular polarized beamsteering antenna array for gigabit wireless communications," IEEE Trans. Antennas and Propagation, vol. 54, no. 2, pp. 743-746, Feb. 2006.

[4] S. Sugiura, S. Chen and L. Hanzo, "Coherent and differential space-time shift keying: A dispersion matrix approach," IEEE Trans. Communications, vol. 58, no. 11, pp. 3219-3230, 2010.

[5] S. Sugiura, S. Chen, H. Haas, P. M. Grant, and L. Hanzo, "Coherent versus non-coherent decode-and-forward relaying aided cooperative space-time shift keying," IEEE Trans. Communications, vol. 59, no. 6, pp. 1707-1719, 2011.

[6] B. Hassibi and B. M. Hochwald, "Cayley differential unitary space-time codes," IEEE Trans. Information Theory, vol. 48, no. 6, pp. 1485-1503, 2002 .

[7] M. Biguesh and A. B. Gershman, "Training-based MIMO channel estimation: A study of estimator tradeoffs and optimal training signals," IEEE Trans. Signal Processing, vol. 54, no. 3, pp. 884-893, 2006.

[8] M. Jiang, J. Akhtman, and L. Hanzo, "Iterative joint channel estimation and multi-user detection for multiple-antenna aided OFDM systems," IEEE Trans. Wireless Communications, vol. 6, no. 8, pp. 2904-2914, 2007.

[9] P. S. Rossi and R. R. Müller, "Joint twofold-iterative channel estimation and multiuser detection for MIMO-OFDM systems," IEEE Trans. Wireless Communications, vol. 7, no. 11, pp. 4719-4729, 2008.

[10] J. Ylioinas and M. Juntti, "Iterative joint detection, decoding, and channel estimation in turbo-coded MIMO-OFDM," IEEE Trans. Vehicular Technology, vol. 58, no. 4, pp. 1784-1796, 2009.

[11] J. Zhang, S. Chen, X. Mu, and L. Hanzo, "Joint channel estimation and multi-user detection for SDMA/OFDM based on dual repeated weighted boosting search," IEEE Trans. Vehicular Technology, vol. 60, no. 7, pp. 3265-3275, 2011.

[12] J. Zhang, S. Chen, X. Mu, and L. Hanzo, "Turbo multi-user detection for OFDM/SDMA systems relying on differential evolution aided iterative channel estimation," IEEE Trans. Communications, vol. 60, no.6, pp.1621-1633, 2012.

[13] Y. Wu, X. Zhu, and A. K. Nandi, "Soft-input turbo channel estimation for single-carrier multiple-input multiple-output systems," IEEE Trans. Vehicular Technology, vol. 58, no. 7, pp. 3867-3873, 2009.

[14] M. Sandell, C. Luschi, P. Strauch, and R. Yan, "Iterative channel estimation using soft decision feedback," in Proc. Globecom 1998 (Sydney, Australia), Nov.8-12, 1998, pp. 3728-3733.

[15] S. Song, A. C. Singer, and K.-M. Sung, "Soft input channel estimation for turbo equalization," IEEE Trans. Signal Processing, vol. 52, no. 10, pp. 2885-2894, 2004.

[16] M. Qaisrani and S. Lambotharan, "An iterative (turbo) channel estimation and symbol detection technique for doubly selective channels," in Proc. VTC2007-Spring (Dublin, Ireland), April 22-25, 2007, pp. 22532256.

[17] L. Hanzo, O. R. Alamri, M. El-Hajjar, and N. Wu, Near-Capacity Multi-Functional MIMO Systems: Sphere-Packing, Iterative Detection and Cooperation. John Wiley \& Sons: Chichester, UK, 2009.

[18] C. Xu, S. Sugiura, S. X. Ng, and L. Hanzo, "Spatial modulation and space-time shift keying: optimal performance at a reduced detection complexity," IEEE Trans. Communications (in press), 2012.

[19] Z. Guo and P. Nilsson, "Algorithm and implementation of the Kbest sphere decoding for MIMO detection," IEEE J. Selected Areas in Communications, vol. 24, no. 3, pp. 491-503, 2006.

[20] L. Wang, L. Xu, S. Chen, and L. Hanzo, "Apriori-LLR-threshold-assisted K-best sphere detection for MIMO channels ," in Proc. VTC2008-Spring (Singapore), May 11-14, 2008, pp. 867-871.

[21] M. Sellathurai and S. Haykin, "Turbo-BLAST for wireless communications: theory and experiments," IEEE Trans. Signal Processing, vol. 50, no. 10, pp. 2538-2546, 2002.

[22] S. M. Kay, Fundamentals of Statistical Signal Processing: Estimation Theory. Englewood Cliffs, NJ: Prentice-Hall, 1993.

[23] A. Scherb, V. Kühn, and K. D. Kammeyer, "Cramer-Rao lower bound for semiblind channel estimation with respect to coded and uncoded finite-alphabet signals," in Proc. 38th Asilomar Conf. Signals, Systems, and Computers (Pacific Grove USA), Nov. 7-10, 2004, pp. 2193-2197. 